\title{
New Dedicated Measuring Devices for Intra-Palm Propagation Signals
}

\author{
Isao Nakanishi, Takashi Inada, and Shigang Li \\ Graduate School of Engineering \\ Tottori University \\ 4-101 Koyama-minami, Tottori, 680-8552 Japan \\ Email: \{D14T2003@faraday.\},\{nakanishi@, li@\}ele.tottori-u.ac.jp
}

\begin{abstract}
We investigated the use of intra-palm propagation signals as biometrics. The intra-palm propagation signal is an electromagnetic wave that is propagated in the shallow part of the skin of a palm. However, the use of a support vector machine (SVM), which is a powerful machine learning method, does not enable superior verification performance. In this paper, we focus on problems related to measuring devices. The first problem is the contact stability between the electrodes and a palm, the second problem is the variation of the electrode position on a palm, and the third problem is the size of a palm. We develop new measuring devices by considering these problems and perform experiments to evaluate their effects.
\end{abstract}

Keywords-biometrics; intra-palm propagation signal; and dedicated measuring device;

\section{INTRODUCTION}

In the case of the user management of systems, successive authentication, such as continuous authentication [1] or ondemand authentication [2], wherein users are required to successively present their biometric data, is required because one-time-only authentication is not capable of preventing identity fraud. Therefore, the password and the identification (ID) card are inapplicable, and only biometric authentication is applicable. Biometric traits that enable the unconscious (transparent) presentation of biometric data are suitable.

The face and the ear are nominated as candidates for this transparent biometrics; however, their data can be insidiously captured by others. This fact enables hackers to produce fakes, which can be used to impersonate genuine users. We confirmed that a face authentication system accepted our faces, which were printed on paper.

Thus, we have proposed the use of intra-body propagation signals as biometrics [3], [4], [5]. An intra-body propagation signal is a signal that is propagated on the skin surface. Because the body composition of people differs, the characteristics of the propagated signal also differ. Because intra-body propagation signals are not exposed on the body surface, they are not easily extracted without being noticed. Thus, the intrabody propagation signal may be useful as a new biometric trait.

In conventional studies [3], [4], [5], signals propagated on forearms were measured and their verification performance was evaluated. However, the usability of measuring signals on forearms is not satisfactory.

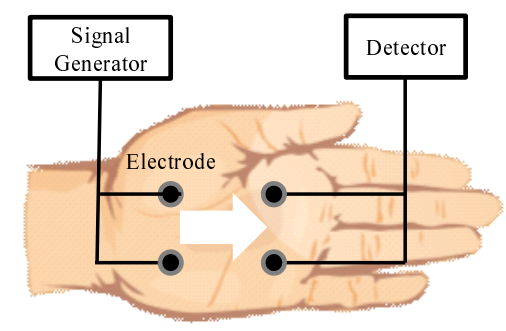

Fig. 1. Measurement of intra-palm propagation signals.

Considering applications for user management, users can control a system while gripping or touching part of the system, such as a handle of a vehicle or a mouse device of a computer. In this situation, palms serve as an interface between the system and the user. We have also examined intra-palm propagation signals [6], [7], which exhibit a low verification performance.

Several issues remain ambiguous. One issue involves dedicated measuring devices for intra-palm propagation signals. Regarding conventional devices, the influence from the contact stability between electrodes and a palm, the variation in electrode position on a palm, and the size of palms were not considered. In this paper, we develop new measuring devices, wherein these aspects are considered and experiments are performed to evaluate their effects.

In Sect. II, conventional studies of person verification using intra-palm propagation signals are explained. In Sect. III, new measuring devices are presented and their effectiveness is evaluated. Concluding remarks are presented in Sect. IV.

\section{PERSON VERIFICATION USING INTRA-PALM PROPAGATION SigNALS}

In this section, we introduce a conventional approach to person verification using intra-palm propagation signals. For details, please refer to the Refs. [6], [7].

\section{A. Intra-Palm Propagation Signal}

A principled structure for measuring intra-palm propagation signals is shown in Fig. 1. A signal from the signal generator is flowed through a pair of electrodes. An electric field is generated around the electrodes in the shallow part of the skin of a palm, and is subsequently stretched to another pair of 


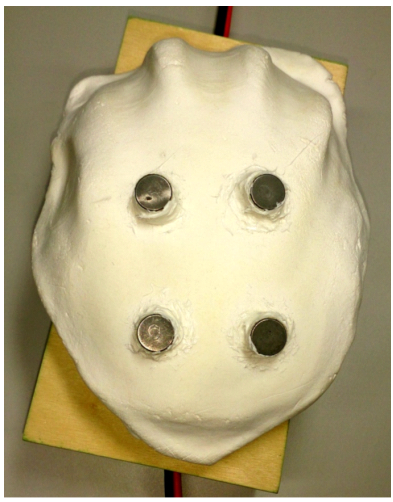

(a)

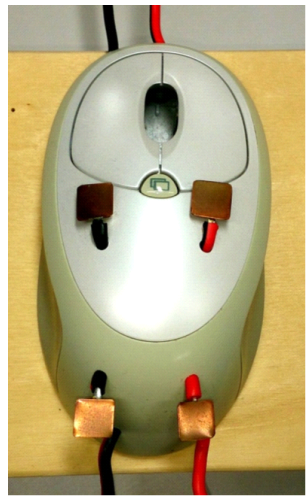

(b)
Fig. 2. Dedicated measuring devices for intra-palm propagation signals.

electrodes; it is detected as a propagated signal by the receiver. We refer to the detected signal as the intra-palm propagation signal.

\section{B. Dedicated Measuring Devices}

To measure intra-palm propagation signals, we prepared dedicated measuring devices, as shown in Fig. 2. (a) is created by making a plaster cast of a palm, and the electrodes are diverted from the metal $(\mathrm{Ag} / \mathrm{AgCl})$ part of commercially produced disposal electrodes. The alignment of the electrodes consists of a width of $2 \mathrm{~cm}$ and a height of $3 \mathrm{~cm}$. The base of (b) is a mouse device for the computer, and cupper plates are used as electrodes. The alignment of the electrodes consists of a width of $2 \mathrm{~cm}$ and a height of $5 \mathrm{~cm}$.

\section{Measurement}

Using the dedicated measuring devices, we measured intrapalm propagation signals from 21 experimental subjects [7]. They sat on a chair and washed the stain from their palms. They put their palms on the dedicated measuring devices on and remained still. A pseudo white noise of $4 \mathrm{~V}_{p-p}$ and $100 \mathrm{MHz}$ bandwidth was output from the signal generator, intra-palm propagation signals were detected by the digital oscilloscope, of which the sampling rate was $1 \mathrm{G}$ samples/s, and the signals were saved in a computer that was connected to the oscilloscope. The measurement was performed two times per day and repeated 30 times (days).

A measurement scene is shown in Fig. 3.

\section{Feature Extraction}

We employed the amplitude spectrum of an intra-palm propagation signal as an individual feature. However, the spectrum is averaged and normalized to suppress intra-individual variation.

The averaging is achieved as follows: the saved data of a detected intra-palm propagation signal are equally divided into several parts, an amplitude spectrum is calculated from each part using a fast Fourier transform (FFT), and an averaged

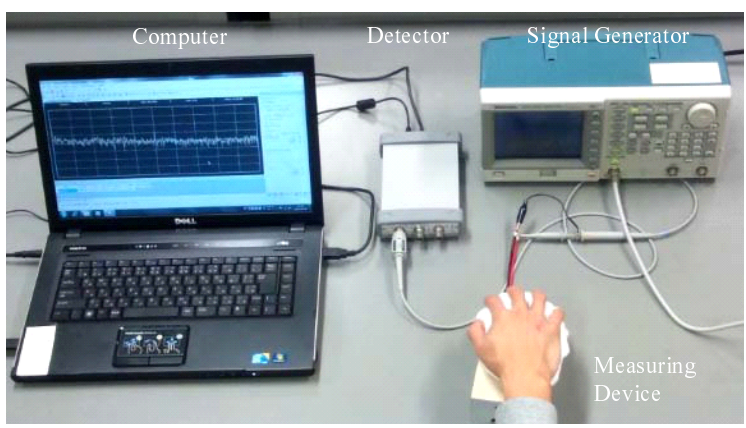

Fig. 3. A measurement scene using the dedicated measuring device.

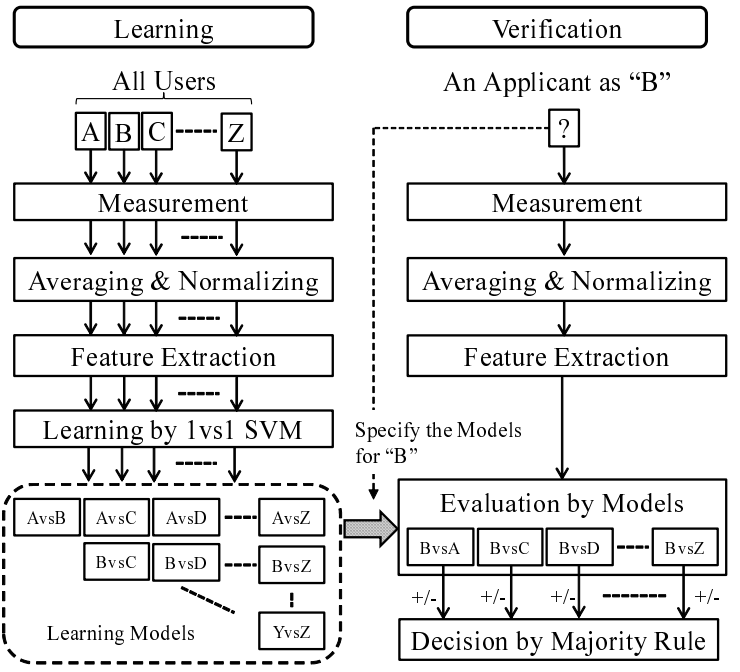

Fig. 4. Verification procedure based on a 1vs1SVM.

spectrum is obtained by ensemble-averaging all amplitude spectra.

The normalizing is achieved by equalizing the means of amplitude spectra from all users.

\section{E. Verification}

First, we evaluated the verification performance on the basis of the Euclidean distance in Ref. [6]. However, the performance was not up to the mark.

Next, we introduced a support vector machine (SVM) into the verification stage [7]. The SVM is a strong classifier based on learning, of which the advantage over other classifiers, such as neural networks, is that the SVM has no local minimum problem [8].

Because a SVM is a two-class classifier, an ingenious scheme is required when the SVM is applied to multi-class classification. We used one versus one (1vs1) SVM, which constructs a learning model that compares a genuine user with another (another) user [9].

Figure 4 shows the verification procedure based on a 1vs1SVM.

SVM models for all users of a system are constructed in the learning stage. Each model is learned by teaching to output 
" +1 " for the intra-palm propagation spectra of a genuine user and " 1 " for the intra-palm propagation spectra of another user.

In the verification stage, an applicant of the system presents a genuine user's name, and his/her intra-palm propagation signal is measured one time. After smoothing, normalizing, and feature extracting, his/her intra-palm propagation spectrum is evaluated in learned models that are related to the specified genuine user. If the number of leaned models that output positive values is larger than a threshold, that is, based on the majority rule, the spectrum is considered to belong to the genuine user, and the applicant is accepted in the system.

\section{F. Performance Evaluation}

For each experimental subject, 40 data (spectra) were employed for the learning of models; the remaining 20 data were used for verification.

The spectral amplitudes for 100 frequency bins (dimensions) were obtained by an FFT and equally divided into 10 parts. Ten amplitude spectral values in the optimal part for each user (subject) were used as an individual feature. The optimal part for each user and the optimal parameters for learning each SVM model were determined using grid searching (round-robin formula).

As a result, the equal error rate (EER) was approximately $24 \%$ regardless of the type of measuring device.

\section{Re-EXAmination of MeAsuring Device}

As presented in the previous section, the SVM verification rate is insufficient. Thus, all processes related to verification must be re-examined. In this paper, we focus on the measuring device. Some issues regarding conventional devices had not been considered.

\section{A. Issues in Conventional Devices}

The first issue is the contact stability between the electrodes and the palm. We used flat-plate types of electrodes, as shown in Fig. 2. However, from the viewpoint of contact stability, point contact may yield better stability than surface contact. Using electrodes that are in point contact with palms may improve the verification performance.

The second issue is the variation in the electrode position on the palm. A guide for placing palms on conventional devices does not exist. The variation in electrode position on palms may be attributed to the lack of guides. Some guides may reduce the variation and improve the verification performance.

The third issue is the size of the palms. The alignment of electrodes was fixed in each conventional device. However, the palm size differs among people. A fixed alignment may be suitable for some subjects but unsuitable for others. Measuring devices wherein the size is fit to the size of the palms may effectively improve the verification performance.

\section{B. New Measuring Devices}

We developed two types of measuring devices, as shown in Fig. 5. The bases of these devices are composed of silicon, and the shape of the palm is concavely modeled.

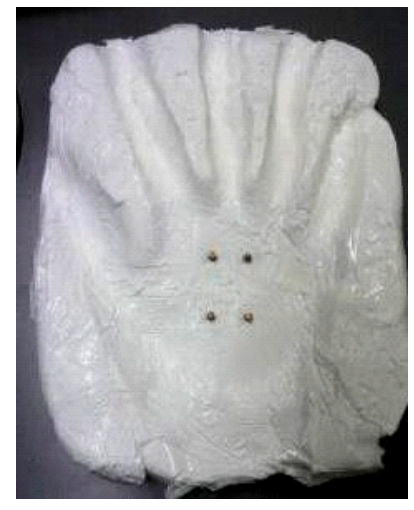

(a) Without guide

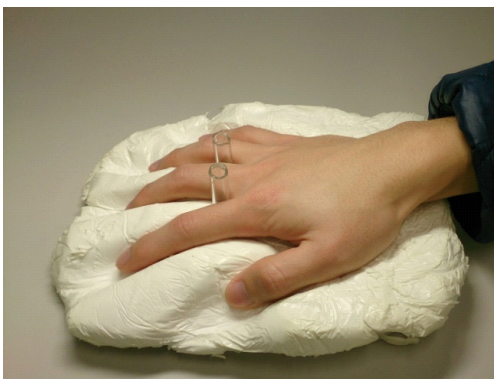

(c) A palm with guide

Fig. 5. New dedicated measuring devices.

The first issue is resolved using copper rods for the electrode instead of cupper plates.

The second issue is expected to be resolved by installing guides on the device. As shown in (c), one acrylic rod is positioned on the fork between the first and second finger, and another rod is positioned between the second and third finger.

The third issue is resolved by preparing three devices with different sizes: large, medium, and small devices. One device is selected according to the palm size of the user.

\section{Comparison of Performance}

To examine the new measuring devices, we measured intrapalm propagation signals using the devices and evaluated their verification performance based on the Euclidean distance.

The number of experimental subjects was 11. Sixty data were obtained from each subject. All processes for verification were identical to the processes in II.

In addition, we adopted the leave one out (LOO) method in this evaluation. Only one subject is excluded, and the performance for the remaining users is evaluated. Although the LOO method is simple, it is generally used to evaluate the influence on performance by specific users.

The EERs are shown in Table I, wherein "None" represents the case in which the LOO method is not employed, that is, 
TABLE I

PERFormance EVALUATION FOR THE LOO METHOD.

\begin{tabular}{c|c|c|c|c}
\hline Excluded Subject & (a) & (b) & Without Guide & With Guide \\
\hline A & 50.2 & 48.7 & 47.9 & 46.6 \\
B & 50.1 & 48.9 & 47.7 & 46.4 \\
C & 50.0 & 48.4 & 48.2 & 47.0 \\
D & 49.8 & 48.7 & 48.2 & 47.6 \\
E & 50.0 & 48.8 & 47.8 & 47.1 \\
F & 50.2 & 48.7 & 47.7 & 46.7 \\
G & 50.2 & 49.3 & 47.8 & 47.7 \\
H & 50.0 & 48.4 & 47.1 & 46.0 \\
I & 50.0 & 49.1 & 48.6 & 47.8 \\
J & 50.0 & 48.7 & 47.5 & 47.1 \\
K & 50.0 & 48.6 & 48.2 & 46.7 \\
\hline None & 49.9 & 48.7 & 47.9 & 46.7
\end{tabular}

using all subjects. For the comparison, conventional devices (a) and (b) in Fig. 2, and new devices without guides are also evaluated.

First, the EERs of each device are equivalent; therefore, the verification performance is not influenced by specific subjects, that is, the size of a palm.

Second, the new devices, which use cupper rods as electrodes, exhibited slightly better performance than the conventional devices.

Third, the point contact type is superior to the surface contact type.

Last, the comparison of the device with a guide with the device without a guide indicated that the EERs are improved by approximately $1 \%$. Therefore, a guide is necessary for measuring intra-palm propagation signals.

Because the purpose of these comparisons is to evaluate the effect of the new measuring devices, we used the Euclidean distance for verification, which is simple and not very powerful. However, it was sufficient to assess the differences between the devices.

\section{CONCLusions}

We have investigated the use of intra-palm propagation signals as biometrics. However, the verification performance of the conventional approach was not superior. Although various issues remain unaddressed, we focused on dedicated devices for measuring the signals. We developed new measuring devices, which contain the following features.

- electrodes have point contact with palms

- guides for stabilizing electrode positions on a palm

- three different sizes for addressing different palm sizes

Using the new devices, we measured intra-palm propagation signals from 11 subjects and verified the performance based on the Euclidean distance. As a result, all modifications of the devices in this paper were effective for improving the verification performance of person authentication using intrapalm propagation signals.

Increasing the number of experimental subjects to obtain reliable results is problematic. The evaluation of the verification performance based on SVM will continue to be challenging.

\section{REFERENCES}

[1] A. Altinok and M. Turk, "Temporal Integration for Continuous Multimodal Biometrics," Proc. of 2003 Workshop on Multimodal User Authentication, pp. 207-214, Dec. 2003.

[2] I. Nakanishi, S. Baba, and C. Miyamoto, "On-Demand Biometric Authentication of Computer Users Using Brain Waves," in Zavoral F. et al. (Eds.), NDT2010, Part I, CCIS 87, Springer, pp. 504-514, Jul. 2010.

[3] I. Nakanishi, Y. Yorikane, Y. Itoh, and Y. Fukui, "Biometric Identity Verification Using Intra-Body Propagation Signal," Proc. of 2007 Biometrics Symposium, Sep. 2007.

[4] I. Nakanishi and Y. Sodani, "SVM-Based Biometric Authentication Using Intra-Body Propagation Signals," Proc. of 2010 7th IEEE International Conference on Advanced Video and Signal Based Surveillance (AVSS2010), pp. 561-566, Sep. 2010.

[5] I. Nakanishi, Y. Sodani, and S. Li, "User Verification Based on the Support Vector Machine Using Intra-Body Propagation Signals,” International Journal of Biometrics, vol. 5, nos. 3/4, pp.288-305, 2013

[6] T. Inada, I. Nakanishi, Y. Sodani, and S. Li, "Biometric Authentication Using Intra-Palm Propagation Signals,” Proc. of International Technical Conference on Circuits/Systems, Computers and Communications (ITCCSCC2012), Jul. 2012.

[7] I. Nakanishi, T. Inada, and S. Li, "Performance Evaluation of IntraPalm Propagation Signals as Biometrics," Proc. of 2013 International Conference on Biometrics and Kansei Engineering (ICBAKE2013), pp. 91-94, Jul. 2013.

[8] N. Cristianini and J. Shawe-Taylor, An Introduction to Support Vector Machines and other kernel-based learning methods, Cambridge University Press, 2000.

[9] C. Hsu and C. Lin, "A Comparison of Methods for Multiclass Support Vector Machines," IEEE Trans. Neural Networks, vol. 13, no.2, pp. 415425, Mar. 2002. 